\title{
Rigidity of glasses and jamming systems at low temperatures
}

\author{
Hajime Yoshino
}

\author{
Department of Earth and Space Science, School of Science, Osaka University, Toyonaka 560-0043, Japan.
}

\begin{abstract}
We discuss a microscopic scheme to compute the rigidity of glasses or the plateau modulus of supercooled liquids by twisting replicated liquids. We first summarize the method in the case of harmonic glasses with analytic potentials. Then we discuss how it can be extended to the case of repulsive contact systems : the hard sphere glass and related systems with repulsive contact potentials which enable the jamming transition at zero temperature. For the repulsive contact systems we find entropic rigidity which behaves similarly as the pressure in the low temperature limit: it is proportional to the temperature $T$ and tends to diverge approaching the jamming density $\phi_{\mathrm{J}}$ with increasing volume fraction $\phi$ as $\lim _{T \rightarrow 0} \mu / T \propto 1 /\left(\phi_{\mathrm{J}}-\phi\right)$, which may account for experimental observations of rigidities of repulsive colloids and emulsions.
\end{abstract}

Keywords: Glass, Jamming, Elasticity

PACS: 61.43.Fs,61.43-j,62.20.D-,64.70.pv,64.70.Q-,83.80.Ab,83.80.Hj,83.80.Iz

\section{INTRODUCTION}

Supercooled liquids, glasses and jamming systems exhibit rich visco-elasticity [1]: at shorter time scales called as the $\beta$-regime such a system behaves as a solid with finite rigidity while it behaves as a liquid with high viscosity at longer time scales called as the $\alpha$-regime. These features appear clearly in the relaxation of shear-stress (see Fig. 1) which follows after switching on a small shear-strain $\gamma$ (See Fig. 2). Approaching the glass transition point the separation of the time scales between the two regimes become enormous so that a supercooled liquid behaves essentially as a quasi-static solid for a very long time. The shear-modulus or the rigidity is the most basic quasi-static property which distinguishes solids from other states of matters.

Among the various types of glasses a class of systems like densely packed repulsive colloids, emulsions, foams and granular particles [2] exhibit an interesting common feature called as the jamming transition: the characters of the amorphous solid state change around the so called jamming point at a certain volume fraction $\phi_{\mathrm{J}}$ at zero temperature. This is manifested in various quasi-static as well as certain dynamic properties of such amorphous solids $[3,4,5,6,7,8,9,10,11,12,13,14,15,16,17$, $18,19,20,21,22,23]$.

The wide time separation between the two regimes suggest that the quasi-static responses in the $\beta$-regime may be analyzed by some statistical mechanical approaches such as the cloned liquid approach which combines the replica method and liquid theory [24, 25, 13, 26]. The latter is a first principle, microscopic approach within the framework of the so called random first order transition (RFOT) theory [27, 28]. Indeed we showed recently that the replica method provides a trick to disentangle the $\beta$ and $\alpha$-like responses and extract the quasi-

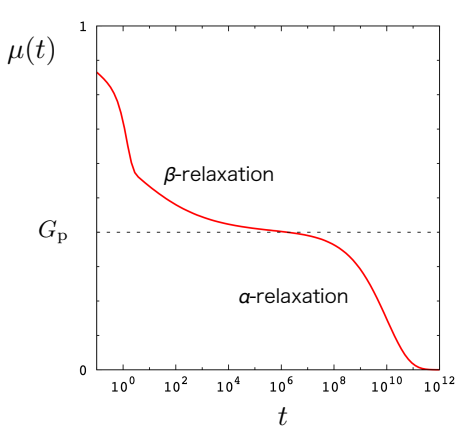

FIGURE 1. Schematic picture of stress relaxation in supercooled liquids. A simplest protocol is considered: switch on the shear-strain of amplitude $\gamma$ (See Fig. 2) at time $t=0$ and measure the relaxation of shear-stress $\sigma(t)$ which follows. Here we define $\mu(t)=\sigma(t) / \gamma$. The plateau value $G_{\mathrm{p}}$ of $\mu(t)$ is called as the plateau modulus which represents the effective rigidity or shear-modulus of metastable glassy states.

static part of the responses in supercooled liquids and structural glasses $[29,30]$.

The purpose of the the present paper is two fold: we first review the method [29, 30] developed for systems with analytic potentials such as the Lennard-Jones potential which are suited for usual molecular glasses. These systems behave as harmonic solids at low temperatures, i. e. systems of random spring networks. Then we attempt to extend the method to account for systems which exhibit the jamming transition. The essential ingredient in such systems is the repulsive contact potentials such as hard-spheres and some soft repulsive contact potentials which are not analytic. Based on this method we analyze the behaviour of the rigidity of hard-spheres and soft repulsive contact systems in the low temperature limit approaching the jamming density $\phi_{\mathrm{J}}$ from below. It appears 
that our result accounts for some experimental observations of the rigidity of repulsive colloids and emulsions $[4,5,6]$.

The organization of the paper is as follows. In the next section we introduce the two distinct classes of systems: systems with the analytic potentials and the repulsive contact potentials. In the subsequent sections we review our strategy $[29,30]$ to extract the quasi-static response functions of supercooled liquids and glasses based on the cloned liquid approach. Then we review the basic fluctuation formulae of the rigidity and our previous scheme to compute the rigidity of harmonic glassy systems reported in $[29,30]$. Finally we discuss the extension of the method to the cases of repulsive contact potentials and analyze how the jamming transition is reflected on the entropic rigidity approaching $\phi_{\mathrm{J}}$.

\section{MODELS}

We consider a generic system of $N$ particles ( $i=$ $1,2, \ldots, N)$ in the 3 -dimensional space with volume $V$ interacting with each other through a two-body potential $v(r)$ which only depends on the relative distance $r$ between particles. The potential part of the Hamiltonian can be written as,

$$
U=\sum_{\langle i j\rangle} v\left(r_{i j}\right) \quad r_{i j}=\left|\mathbf{r}_{i}-\mathbf{r}_{j}\right|
$$

where $\langle i j\rangle$ stands for summation over the $N(N-1) / 2$ pairs of the particles and $\mathbf{r}_{i}(i=1,2, \ldots, N)$ represents the position of the particles. We suppose that the temperature $T$ is low enough and the number density $\rho=N / V$ is high enough such that the system is in a supercooled liquid or a glassy metastable state.

We consider two distinct classes of systems:

- Harmonic systems: the potential $v(r)$ is an analytic function of $r$ for $r>0$ like the Lennard-Jones potential. Presumably this class of systems is relevant for molecular glasses.

For an explicit model computation we consider the soft-core potential $v(r)=\varepsilon(r / a)^{-12}$ where $\varepsilon$ and $a$ are the unit energy and length respectively.

- Repulsive contact systems : the potential $v(r)$ is repulsive and has a definite cut-off at the scale the particle size $a$ like the hard-spheres. Presumably this class of systems is relevant for emulsions and repulsive colloids. The density is a crucial parameter in these systems and it is convenient to represent it via volume fraction $\phi$ which is related to the number density $\rho$ as $\phi=(\pi / 6) \sigma^{3} \rho$. An important feature of this class of systems is that they exhibit the jamming transition at $T=0$ by increasing the volume fraction $\phi$ up to some jamming density $\phi_{\mathrm{J}}$.
For an explicit model computation we consider the soft-particle potential $v(r)=\varepsilon(1-r / a)^{2} \theta(1-r / a)$ where $\theta(r)$ is the step function. In the present paper we limit ourselves to the range of volume fractions $\phi<\phi_{\mathrm{J}}$ where the system behaves as hard-spheres in the zero temperature limit $T \rightarrow 0$.

\section{DISENTANGLEMENT OF THE INTRA-STATE AND INTER-STATE RESPONSES}

\subsection{A mean-field picture: ensemble of metastable states}

Let us take the basic energy landscape picture [31, 32] : we consider that the equilibrium state of a supercooled liquid or a glass can be described in terms of a statistical ensemble of metastable states, which might be interpreted as metabasins [32] each of which is a union of inherent structures [31]. Let us label the metastable states as $\alpha=1,2,3, \ldots$ and denote the free-energy per particle of the $\alpha$-th state as $f_{\alpha}$. Then the equilibrium free-energy of the system may be expressed formally as,

$$
\begin{gathered}
F(h)=-k_{\mathrm{B}} T \log Z(h) \\
Z(h) \simeq \sum_{\alpha} e^{-N \beta f_{\alpha}(h)}
\end{gathered}
$$

where $k_{\mathrm{B}}$ is the Boltzmann's constant and $\beta \equiv 1 / k_{\mathrm{B}} T$.

We have introduced a parameter $h$ which represents a generic infinitesimal probing field, such as the shear which we will focus on in the present paper. The linear susceptibility to the external field $h$ can be seen to take the following generic form,

$$
\left.\chi \equiv \frac{1}{N} \frac{d^{2} F(h)}{d h^{2}}\right|_{h=0}=\hat{\chi}+\tilde{\chi}
$$

with

$$
\hat{\chi}=\left[\left[\chi_{\alpha}\right]\right] \quad \tilde{\chi}=\beta N\left(\left[\left[o_{\alpha}^{2}\right]\right]-\left[\left[o_{\alpha}\right]\right]^{2}\right)
$$

Here $o_{\alpha} \equiv d f_{\alpha}(h) /\left.d h\right|_{h=0}$ is the equilibrium value of an observable $o$ which is conjugated to the external field $h$. Similarly $\chi_{\alpha} \equiv d o_{\alpha} / d h=d^{2} f_{\alpha}(h) /\left.d h^{2}\right|_{h=0}$ is the associated linear susceptibility within a given metastable state $\alpha$. In (4) [...]] stands for averaging over the ensemble of the metastable states defined as,

$$
\left[[\ldots] \equiv \frac{\sum_{\alpha} e^{-N \beta f_{\alpha}(0)} \ldots}{Z(0)} .\right.
$$

The important feature evident in (3) is that the total linear susceptibility $\chi$ is the sum of two distinct parts: $\hat{\chi}$ and 
$\tilde{\chi}$ associated with the response within metastable states and response due to jumps between different metastable states. Physically $\hat{\chi}$ can be regarded as the quasi-static response within the $\beta$-regime and $\tilde{\chi}$ can be related to the response in the $\alpha$-regime. Although the two parts have very different characters, (3) implies they are mixed up in the total response. We wish to disentangle the two. Let us discuss below how the replica trick works for this purpose.

\subsection{Response of a cloned system}

Let us consider a cloned system [33, 34, 24] which consists of $m$ replicas of the same system labeled as $a=1,2, \ldots, m$. The free-energy of the cloned system is defined as,

$$
\begin{array}{r}
F_{m}\left(\left\{h_{a}\right\}\right)=-\frac{k_{\mathrm{B}} T}{m} \log Z_{m}\left(\left\{h_{a}\right\}\right) \\
Z_{m}\left(\left\{h_{a}\right\}\right) \simeq \sum_{\alpha} e^{-N \beta \sum_{a=1}^{m} f_{\alpha}\left(h_{a}\right)}
\end{array}
$$

Note that there is only one summation over the metastable states instead of $m$ summations. This means that we are assuming that $m$ replicas are not allowed to fluctuate independently from each other but forced to fluctuate together over different metastable states. Yet the replicas are allowed to fluctuate differently from each other within the metastable states. How to realize such a situation in practice is a non-trivial task by itself $[33,34,24,13]$ as we discuss shortly later.

The key point is that we have put different probing fields $h_{a}(a=1,2, \ldots, m)$ on different replicas in (6) [29, 30]. It naturally lead us to define a sort of generalized linear-susceptibility of a matrix form,

$$
\left.\chi_{a b} \equiv \frac{1}{N} \frac{\partial^{2} F_{m}(\{h\})}{\partial h_{a} \partial h_{b}}\right|_{\left\{h_{a}=0\right\}}=\hat{\chi}_{m} \delta_{a b}+\tilde{\chi}_{m}
$$

where $\hat{\chi}_{m}$ and $\tilde{\chi}_{m}$ are almost the same as $\hat{\chi}$ and $\tilde{\chi}$ defined in (4) but evaluated by replacing $[[\ldots]]$ by $[[\ldots]]_{m}$ defined as,

$$
[[\ldots]]_{m} \equiv \frac{\sum_{\alpha} e^{-N m \beta f_{\alpha}(0)}}{Z_{m}(0)}
$$

Quite remarkably the 2nd equation of (7), which can be easily verified, implies that the $\beta$ and $\alpha$-like responses can be distinguished from each other:

$$
\begin{array}{r}
\hat{\chi}=\lim _{m \rightarrow 1} \hat{\chi}_{m} \quad \hat{\chi}_{m}=\chi_{a a}-\chi_{a \neq b} \\
\tilde{\chi}=\lim _{m \rightarrow 1} \tilde{\chi}_{m} \quad \tilde{\chi}_{m}=\chi_{a \neq b}
\end{array}
$$

\subsection{Cloned liquid}

Here let us briefly sketch how to implement a cloned system [24, 13]. The basic idea is to introduce a system of an artificial molecular liquid in which each 'molecule' $i=1,2, \ldots, N$ consists of $m$ particles belonging to different replicas $a=1,2, \ldots, m$. The particles are allowed to fluctuate only within the molecule of size $A$, which is interpreted physically as the cage size. The cage size $A$ is determined by a variational principle (see below). Existence of a solution with a finite cage size $A<\infty$ implies existence of metastable states [34, 24].

The coordinates of the particles $\mathbf{r}_{i}^{a}(i=1,2, \ldots, N)$ can be decomposed formally as,

$$
\mathbf{r}_{i}^{a}=\mathbf{R}_{i}+\mathbf{u}_{i}^{a} \quad \mathbf{R}_{i} \equiv \frac{1}{m} \sum_{a=1}^{m} \mathbf{r}_{i}^{a}
$$

where $\mathbf{u}_{i}^{a}$ stands for fluctuation of the particle belonging to the $a$-th replica with respect to the center of mass $\mathbf{R}_{i}$ of the molecule. The fluctuations within the molecules are assumed to obey the Gaussian statistics with the mean and variance given by [24],

$$
\left\langle\left(\mathbf{u}_{i}^{a}\right)^{\mu}\right\rangle_{\mathrm{cage}}=0 \quad\left\langle\left(\mathbf{u}_{i}^{a}\right)^{\mu}\left(\mathbf{u}_{j}^{b}\right)^{v}\right\rangle_{\mathrm{cage}}=A\left(\delta_{a b}-\frac{1}{m}\right) \delta_{\mu \nu} \delta_{i j}
$$

Here $\mu$ (and $v$ ) represents a component of 3-dimensional vectors $\mu=x, y, z$. The factor $\delta_{a b}-\frac{1}{m}$ reflects the constraint $\sum_{a=1}^{m}\left(\mathbf{u}^{a}\right)=\mathbf{0 Z}$.

The free-energy of the molecular or cloned liquid $G_{m}(A)$ of a given number of replicas $m$ and the cage size $A$ can be obtained as follows [24]. First one integrate out the fluctuations within the molecules which amounts to replace the original interaction potential $v(r)$ by a remornalized one $v_{\mathrm{eff}}(r, A)$. [24, 13] In the case of analytic potentials it reads as [24],

$$
v_{\mathrm{eff}}(r)=v(r)-(1-m) \frac{A}{m^{2}} \nabla^{2} v(r)+\ldots .
$$

Then one is left to integrate out the CM positions of the molecules interacting with each other via $v_{\text {eff }}(r, A)$ and subjected to a heat-bath at an effective temperature $T / \mathrm{m}$. Eventually we have to take the $m \rightarrow 1$ limit (see (10)).

The strategy is to start from sufficiently small $m(\ll$ 1) so that the cloned system remain in the liquid state because the effective temperature $T / m$ becomes sufficiently high even if the actual temperature $T$ itself is very low. Then standard density functional methods of the liquid theory [35] allows one to compute the free-energy $G_{m}(A)$. The value of $A$ is determined by minimizing the variational free-energy $G_{m}(A)$ with respect to $A$ yielding $F_{m}=\min _{A} G_{m}(A)$. Let us denote the value of the cage size $A$ at the minimum as $A^{*}(m, T, \rho)$.

The last step is to take the limit $m \rightarrow 1^{-}$. It turns out that at temperatures $T$ below the ideal glass transition 
temperature, i. e. the Kauzmann temperature $T_{\mathrm{K}}(\rho)$, one finds a characteristic value $m^{*}=m^{*}(T, \rho)$ in the range $0 \leq m^{*}(T, \rho) \leq 1$ such that

$$
\lim _{m \rightarrow 1^{-}} F_{m}(T, \rho)=F_{m^{*}}(T, \rho)
$$

holds. The Kauzmann transition temperature $T_{\mathrm{K}}(\rho)$ can be obtained by solving $m^{*}\left(T_{\mathrm{K}}(\rho), \rho\right)=1$. The reason behind (14) is actually the entropy crisis mechanism, i. e. the ideal glass transition, taking place along the $m$-axis. We refer the readers to Refs [24] for the details. The above observation implies that $\beta$ and $\alpha$-like responses (see (10)) can be obtained as,

$$
\hat{\chi}=\hat{\chi}_{m^{*}} \quad \tilde{\chi}=\tilde{\chi}_{m^{*}}
$$

\section{MICROSCOPIC COMPUTATION OF THE RIGIDITY OF GLASSES}

\subsection{Static linear response to shear}

Now let us study static linear response to shear. For clarity we recall and compare the well known fluctuation formulae for the shear-modulus for analytic potentials [36] and hard-spheres [37] in the case of simple shear. To this end we consider a system of particles $i=1,2, \ldots, N$ put in a rectangular container of volume $V$ and perturb the system by a shear-strain of infinitesimal amplitude $\gamma$ on the container (See Fig. 2). Most important feature of the shear is that it just changes the shape of the container but not the volume $V$ (and thus the density $\rho$ ).

The free-energy $F(\gamma)$ of the system may be formally expanded in power series of $\gamma$ as,

$$
F(\gamma) / V=F(0) / V+\gamma \sigma+\frac{\gamma^{2}}{2} \mu+\ldots
$$

Here the coefficients of the 1 st and 2 nd order terms in the expansion defines the shear-stress $\sigma$ and the rigidity or the shear-modulus $\mu$. Microscopic expressions of $\sigma$ and $\mu$ can be obtained as follows.

The free-energy $F(\gamma)$ can be expressed formally as,

$$
\begin{aligned}
& F(\gamma) \equiv-k_{\mathrm{B}} T \log Z(\gamma) \\
& Z(\gamma) \equiv \int_{\mathscr{V}(\gamma)} \prod_{i=1}^{N} \frac{d^{3} r_{i}}{\lambda_{\mathrm{th}}^{3}} e^{-\beta \sum_{\langle i j\rangle} v\left(r_{i j}\right)} \\
& =\left.\int_{\mathscr{V}(0)} \prod_{i=1}^{N} \frac{d^{3} r_{i}^{\prime}}{\lambda_{\mathrm{th}}^{3}} e^{-\beta \sum_{\langle i j\rangle} v\left(r_{i j}\right)}\right|_{r_{i j}=\sqrt{\left(x_{i j}^{\prime}+\gamma z_{i j}^{\prime}\right)^{2}+\left(y_{i j}^{\prime}\right)^{2}+\left(z_{i j}^{\prime}\right)^{2}}}
\end{aligned}
$$

Here $\lambda_{\text {th }}$ is the thermal de Brogile length. The subscripts $\mathscr{V}(\gamma)$ represent the range of integrations, including not only the volume $V$ (which is invariant under shear) but also its shape, parametrized by the shear-strain $\gamma$. In the 2nd equation we changed the integration variables from $\mathbf{r}$ to $\mathbf{r}^{\prime}$ (See Fig. 2) which allows us to change the integration region $\mathscr{V}(\gamma)$ back to the unperturbed one $\mathscr{V}(0)$. The 2 nd equation allows us to easily obtain the expansion of the free-energy $F(\gamma)$ in power series of $\gamma$.

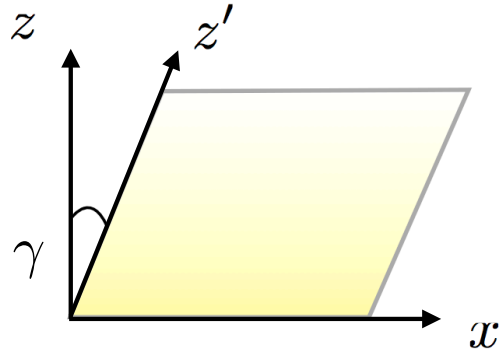

FIGURE 2. The geometry of a sheared container.

Shear-stress. Microscopic expression of the shearstress is obtained as,

$$
\left.\sigma \equiv \frac{1}{V} \frac{\partial F(\gamma)}{\partial \gamma}\right|_{\gamma=0}=\frac{1}{V} \sum_{\langle i j\rangle}\left\langle\sigma\left(\mathbf{r}_{i j}\right)\right\rangle
$$

where $\langle\ldots\rangle$ is the thermal average,

$$
\langle\ldots\rangle \equiv \frac{1}{Z(0)} \int_{\mathscr{V}(0)} \prod_{i=1}^{N} \frac{d^{3} r_{i}^{\prime}}{\lambda_{\mathrm{th}}^{3}} e^{-\beta \sum_{\langle i j\rangle} v\left(r_{i j}\right)} \ldots
$$

Here we introduced the local shear-stress,

$$
\sigma(\mathbf{r}) \equiv \hat{z} \hat{x} r v^{\prime}(r)
$$

with short-hand notations $\hat{x} \equiv x / r, \hat{y} \equiv y / r, \hat{z} \equiv z / r$ where $r=\sqrt{x^{2}+y^{2}+z^{2}}$.

Remark: note that the expression (20) is ill-defined for hard-spheres. However the average shear-stress Eq. (18) can still be evaluated safely by noting that $-\beta v^{\prime}(r) e^{-\beta v(r)}=\left(e^{-\beta v(r)}\right)^{\prime}$ becomes a delta function $\delta(r-a)$ for hard-spheres and soft repulsive contact systems in the $T \rightarrow 0$ limit.

Shear-modulus. Similarly the microscopic expression or the static fluctuation formula of the shearmodulus is obtained as,

$$
\begin{aligned}
& \left.\mu \equiv \frac{1}{V} \frac{\partial^{2} F(\gamma}{\partial \gamma^{2}}\right|_{\gamma=0}=\mu_{\text {born }} \\
& \left.\left.-\beta V \sum_{\langle k l\rangle\langle m n\rangle} \sum_{\left\langle\sigma\left(\mathbf{r}_{k l}\right)\right.} \sigma\left(\mathbf{r}_{m n}\right)\right\rangle-\left\langle\sigma\left(\mathbf{r}_{k l}\right)\right\rangle\left\langle\sigma\left(\mathbf{r}_{m n}\right)\right\rangle\right)
\end{aligned}
$$

where $\mu_{\text {born }}$ is the so called Born term defined as,

$$
\mu_{\text {born }} \equiv \frac{1}{V} \sum_{\langle i j\rangle} \hat{z}^{2}\left[r^{2} v^{\prime \prime}(r) \hat{x}^{2}+r v^{\prime}(r)\left(1-\hat{x}^{2}\right)\right] \text {. }
$$


The Born term $\mu_{\text {born }}$ represents the instantaneous, affine response to shear while the 2 nd term in the r.h.s of the 2nd equation (21) represents the so called non-affine correction due to stress relaxation $[36,30]$.

The above expressions (21) (22) are problematic for the repulsive contact systems. Especially the Born term is formally infinite for hard-spheres. The Born term Eq. (22) stems from direct spatial derivatives of the local stress (20) which does not exist in this class of systems in sharp contrast to the harmonic systems (See the remark below (20)). Then for this class of systems it is more convenient to use an alternative but equivalent expression [37],

$$
\begin{aligned}
& \beta \mu=V\left[\sum_{\langle k l\rangle}\left\langle\beta \sigma\left(\mathbf{r}_{k l}\right)\right\rangle^{2}\right. \\
& \left.-\sum_{\langle k l\rangle} \sum_{\langle m n\rangle \neq\langle k l\rangle}\left(\left\langle\beta \sigma\left(\mathbf{r}_{k l}\right) \beta \sigma\left(\mathbf{r}_{m n}\right)\right\rangle-\left\langle\beta \sigma\left(\mathbf{r}_{k l}\right)\right\rangle\left\langle\beta \sigma\left(\mathbf{r}_{m n}\right)\right\rangle\right)\right]
\end{aligned}
$$

$$
\begin{aligned}
& \hat{\mu}=\mu_{\mathrm{born}}^{\mathrm{eff}} \\
& -\frac{k_{\mathrm{B}} T}{m^{*}}\left(\frac{A^{*}}{m^{*}}\right) \rho \int d^{3} r\left|\left(\beta m^{*} \nabla \sigma(\mathbf{r})\right)\right|^{2} g_{T / m^{*}, \rho}(\mathbf{r}) \\
& -\frac{k_{\mathrm{B}} T}{m^{*}}\left(\frac{A^{*}}{m^{*}}\right) \rho \int d^{3} r_{1} d^{3} r_{2} \\
& \left(\beta m^{*} \nabla \sigma\left(\mathbf{r}_{1}\right)\right) \cdot\left(\beta m^{*} \nabla \sigma\left(\mathbf{r}_{2}\right)\right)\left(g_{3}\right)_{T / m^{*}}\left(\mathbf{r}_{1}, \mathbf{r}_{2}\right)
\end{aligned}
$$

while the $\alpha$-like part of the response is given by

$$
\tilde{\mu}=-\frac{\hat{\mu}}{m^{*}}
$$

because of the sum rule $\sum_{b=1}^{m^{*}} \mu_{a b}=0$ reflecting the plain fact the cloned liquid as a whole is just a liquid. Physically the latter suggests static analogue of the yielding processes (See [30] for discussions). In the above equations $m^{*}=m^{*}(T, \rho)$ and $A^{*}=A^{*}\left(m^{*}(T, \rho), T, \rho\right)$ are the values determined in the course of the evaluation of the free-energy of the cloned liquid discussed before. Here $g_{T, \rho}(r)$ and $\left(g_{3}\right)_{T, \rho}\left(\mathbf{r}_{1}, \mathbf{r}_{2}\right)$ are the radial distribution function and the three-point correlation function of the liquid at temperature $T$ and density $\rho$ respectively.

The term $\mu_{\text {born }}^{\text {eff }}$ represents the Born term (22) associated with the renormalized potential (13), which itself consists of the original born term (22) at $O\left(A^{0}\right)$ and corrections at $O(A)$. The last two terms on the r.h.s of (25) represents the effect of the stress relaxation due to the fluctuation inside the cages, i.e. $\beta$-relaxation (See Fig. 3).

We show the result of a model computation on the binary soft-core system $v(r) \sim(r / a)^{-12}$ in Fig. 4 . The result at finite temperature $T>0$ was already reported in $[29,30]$. Here we added the result of a computation performed directly in the zero temperature limit $T \rightarrow 0$.

The cloned liquid computation can be performed in the zero temperature limit $T \rightarrow 0$ by introducing scaled variables $\alpha=A / T$ and $\tau=T / m$ [14]. The expression of the rigidity $\hat{\mu}$ given Eq. (25) also suggest it has a limiting value in the $T \rightarrow 0$ limit.

For the binary soft-core system we consider $T \rightarrow 0$ limit of the the theory formulated in Ref [25] which where $\mu_{\text {born }}$ and the local shear-stress $\sigma(\mathbf{r})$ are the same as those defined in Eq. (22) and Eq. (20).

Now we switch on the cloning following the prescription discussed previously using the decomposition of the coordinates (11). This allows us to expand the local shear-stress $\sigma\left(\mathbf{r}_{i j}^{a}\right)$ as,

$$
\sigma\left(\mathbf{r}_{i j}^{a}\right)=\sigma\left(\mathbf{R}_{i j}\right)+\left.\nabla \sigma(r)\right|_{r=\mathbf{R}_{i j}} \cdot\left(\mathbf{u}_{i}^{a}-\mathbf{u}_{j}^{a}\right)+\ldots
$$

as well as the interaction potential as,

$$
\begin{aligned}
& v\left(r_{i j}^{a}\right)=v\left(R_{i j}\right)+\left.\nabla v(r)\right|_{r=R_{i j}} \cdot\left(\mathbf{u}_{i}^{a}-\mathbf{u}_{j}^{a}\right) \\
& +\left.\frac{1}{2} \sum_{\mu v} \frac{\partial^{2} v(r)}{\partial r^{\mu} \partial r^{v}}\right|_{r=R_{i j}}\left(\left(u_{i}^{a}\right)^{\mu}-\left(u_{j}^{a}\right)^{\mu}\right)\left(\left(u_{i}^{a}\right)^{v}-\left(u_{j}^{a}\right)^{v}\right)+\ldots
\end{aligned}
$$
matrix in the form of the anticipated matrix structure (7),

$$
\mu_{a b}=\hat{\mu} \delta_{a b}+\tilde{\mu}
$$




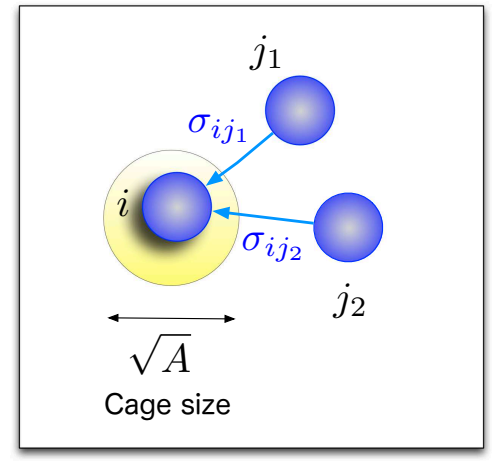

FIGURE 3. Schematic picture of stress relaxation due to motions of particles inside cages. The local motion of the particle $i$ can relax local stress between the particle $i$ and the surrounding particles $j_{1}, j_{2}, \ldots$.

employs the binary HNC approximation for the computation of the liquid free energy and the radial distribution function $g_{T}(r)$. As the result we find the effective temperature of the cloned liquid converges to $\tau \equiv \lim _{T \rightarrow 0} T / m^{*}(T) \simeq 0.115$ within the 1 st order cage expansion. This value is close to the Kauzmann transition temperature $T_{\mathrm{K}} / \varepsilon \sim 0.12$ where $m^{*}\left(T_{\mathrm{K}}\right)=1$ [25].

The result shown in Fig. 4 suggest the rigidity at low temperatures $T<T_{\mathrm{K}}$ behaves essentially as if at $T=0$. Moreover the numerical values of the plateau modulus $G_{\mathrm{p}}$ observed by MD simulations [38, 39], and the value of the rigidity of the inherent structures [40] compare well with the theoretical values shown in Fig. 4. These observations strongly support the usual view that the metastable glassy states at low enough temperatures are harmonic solids which can be efficiently described as systems of random spring network (see also [41] for a related work).

Note also that the rigidity becomes significantly smaller with increasing temperature above $T_{\mathrm{K}}$. We interpret this as reduction of the rigidity [30] due to thermally activated plastic events among a union of inherent structures [31] belongin to a common metabasin [32] or a metastable state. The rigidity apparently vanishes (crosses 0 ) around $T / \varepsilon \sim 0.22$, suggesting melting of metastable states, which happens to be rather close to the so called MCT critical temperature $T_{\mathrm{c}} / \varepsilon \sim 0.19-0.22$ [42][26]. However the first order cage expansion which we have employed does not allow us to locate the MCT transition temperature at which the glassy solution with finite cage size $A<\infty$ disappear presumably by a spinodal like mechanism [34]. As we noted in [30] we rather consider at the moment that, at least in the mean-field sense, the rigidity should exhibit a discontinuous behaviour. This is because the expression (25) actually implies that the rigidity is a function of the the cage size $A$ which is predicted to behave as $A(T)-A\left(T_{\mathrm{c}}\right) \propto$
$\sqrt{T_{\mathrm{c}}-T}$ approaching the dynamical temperature $T_{\mathrm{c}}$ from below by the mode coupling theory (MCT) [43]. Indeed such a discontinuous behaviour of the rigidity has been suggested in an alternative formulation of the replica approach[44].

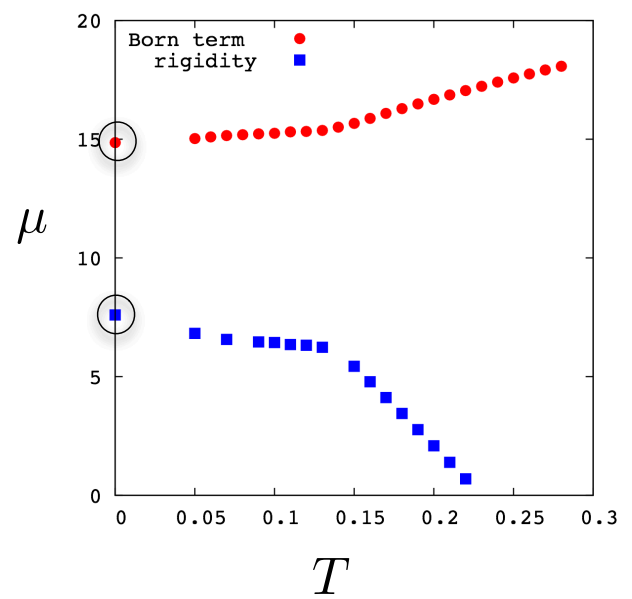

FIGURE 4. The rigidity of a binary soft-core system. The born term which represents the instantaneous, affine response and the rigidity $\hat{\mu}$ which includes non-affine corrections by stress relaxation due to fluctuations inside cages are shown. The results are $T>0$ are reported in $[29,30]$. The Kauzmann temperature is $T_{\mathrm{K}} / \varepsilon \sim 0.12$.

\section{REPULSIVE CONTACT SYSTEMS}

Finally we are in the position to analyze the rigidity of the glassy states of repulsive contact systems: the hardspheres and generic soft repulsive contact systems in the low temperature limit $T \rightarrow 0$.

Again we first consider the rigidity matrix of the 'free' $m$-replica system, which is obtained as,

$$
\begin{aligned}
& \beta \mu_{a b}=V\left[\sum_{\langle k l\rangle}\left\langle\beta \sigma\left(\mathbf{r}_{k l}^{a}\right)\right\rangle\left\langle\beta \sigma_{b}\left(\mathbf{r}_{k l}^{b}\right)\right\rangle\right. \\
& \left.-\sum_{\langle k l\rangle} \sum_{\langle m n\rangle \neq\langle k l\rangle}\left(\left\langle\beta \sigma\left(\mathbf{r}_{k l}^{a}\right) \beta \sigma\left(\mathbf{r}_{m n}^{b}\right)\right\rangle-\left\langle\beta \sigma\left(r_{k l}^{\mathbf{a}}\right)\right\rangle\left\langle\beta \sigma\left(r_{m n}^{b}\right)\right\rangle\right)\right] .
\end{aligned}
$$

As expected it is similar to the expression Eq. (23) for the single system.

Then by switching on the cloning we obtain the rigidity of metastable states (plateau modulus $G_{\mathrm{p}}$ ) up to $O(A)$ as,

$$
\begin{array}{r}
\beta \hat{\mu}=-\frac{1}{m^{*}}\left(\frac{A^{*}}{m^{*}}\right) \frac{6}{\pi} \frac{\phi}{\sigma^{3}} \int d^{3} r_{1} d^{3} r_{2} \\
\left(\nabla \beta m^{*} \sigma\left(\mathbf{r}_{1}\right)\right) \cdot\left(\nabla \beta m^{*} \sigma\left(\mathbf{r}_{2}\right)\right)\left(g_{3}\right)_{T / m *, \phi}\left(\mathbf{r}_{1}, \mathbf{r}_{2}\right)
\end{array}
$$


Most important difference from the case of the harmonic systems (25) is that the Born term is apparently absent here. Consequently the rigidity is 0 at order $O\left(A^{0}\right)$ and starts only at $O(A)$. The relevant term at $O(A)$ is again the one related to the thermal fluctuation of the shearstress due the the fluctuations inside cages (See Fig. 3).

In order to make further progresses, we approximate the three-point correlation function $g_{3}\left(\mathbf{r}_{1}, \mathbf{r}_{2}\right)$ by the Kirkwood approximation,

$$
\left(g_{3}\right)_{T, \phi}\left(\mathbf{r}_{1}, \mathbf{r}_{2}\right) \simeq g_{T, \phi}\left(r_{1}\right) g_{T, \phi}\left(r_{2}\right) g_{T, \phi}\left(\left|\mathbf{r}_{1}-\mathbf{r}_{2}\right|\right) .
$$

Then using the cavity function $y_{T, \phi}(r)$ defined as $g_{T, \phi}(r) \equiv y_{T, \phi}(r) e^{-\beta v(r)}$ we find,

$$
\beta \hat{\mu}=\frac{1}{m^{*}}\left(\frac{A^{*}}{m^{*}}\right) \frac{6}{\pi} \frac{\phi}{\sigma^{3}} C
$$

with

$$
\begin{aligned}
& C \simeq-\int d^{3} r_{1} d^{3} r_{2} y_{T / m^{*}, \phi}\left(\left|\mathbf{r}_{1}-\mathbf{r}_{2}\right|\right) e^{-\beta m^{*} v\left(\left|\mathbf{r}_{1}-\mathbf{r}_{2}\right|\right)} \\
& \nabla_{1}\left(y_{T / m^{*}, \phi}\left(r_{1}\right) \hat{z}_{1} \hat{x}_{1} r_{1} \frac{d e^{-\beta m^{*} v\left(r_{1}\right)}}{d r_{1}}\right) \\
& \cdot \nabla_{2}\left(y_{T / m^{*}, \phi}\left(r_{2}\right) \hat{z}_{2} \hat{x}_{2} r_{2} \frac{d e^{-\beta m^{*} v\left(r_{2}\right)}}{d r_{2}}\right) \\
& \underset{T \rightarrow 0}{\longrightarrow}-\left(y_{\phi}^{\mathrm{HS}}(a)\right)^{2} \int d \Omega_{1} d \Omega_{2} \int d r_{1} d r_{2} r_{1}^{2} r_{2}^{2} \\
& y_{\phi}^{\mathrm{HS}}\left(\left|\mathbf{r}_{1}-\mathbf{r}_{2}\right|\right) \theta\left(\left|\mathbf{r}_{1}-\mathbf{r}_{2}\right|-a\right) \\
& \nabla_{1}\left(\hat{z}_{1} \hat{x}_{1} r_{1} \delta\left(r_{1}-a\right)\right) \cdot \nabla_{2}\left(\hat{z}_{2} \hat{x}_{2} r_{2} \delta\left(r_{2}-a\right)\right)
\end{aligned}
$$

In the last equation we took $T \rightarrow 0$ limit which greatly simplifies the calculation. The evaluations of the integrals over the polar coordinates are tedious but straight forward. First the integrations along the radial coordinates $r_{1}$ and $r_{2}$ can be done exactly via integrations by parts. Subsequently the integrations over the solid angles $\Omega_{1}$ and $\Omega_{2}$ can be simplified, with the help of spherical harmonics, to simple one dimensional integrals over $x=\cos \left(\theta_{12}\right)$ where $\theta_{12}$ is the relative angle between the two solid angles. If we make a further approximation $y_{\phi}^{\mathrm{HS}}\left(\left|\mathbf{r}_{1}-\mathbf{r}_{2}\right|\right) \simeq y_{\phi}^{\mathrm{HS}}(a)$ the last integrations can also be done exactly and we finally obtain,

$$
C \simeq\left(y_{\phi}^{\mathrm{HS}}(a)\right)^{3} \frac{113}{120} \pi^{2} .
$$

The above results suggest finite rigidity $\hat{\mu}$ of the repulsive contact systems which is proportional to the temperature $T$ meaning that it is of entropic origin in sharp contrast to the harmonic systems discussed previously whose rigidity is essentially mechanical.

In order to study how the rigidity depends on the volume fraction $\phi$, we need to know the values of $m^{*}(T=$
$0, \phi), A^{*}(T=0, \phi)$ and $y_{\phi}^{\mathrm{HS}}(a)$. Fortunately they are provided by recent studies on the hard-sphere glass [13] and a soft repulsive contact potential system [14] close to the jamming density, more precisely the so called glass close packing density $\phi_{\mathrm{GCP}}$. According to the latter works $m^{*}(T=0, \phi) \simeq c_{1}\left(\phi_{\mathrm{GCP}}-\phi\right)$ and $A^{*}(T=0, \phi) \simeq$ $c_{2}\left(\phi_{\mathrm{GCP}}-\phi\right)$ approaching $\phi_{\mathrm{GCP}}$ from below with $c_{1}$ and $c_{2}$ being some positive constants. The value of $y_{\phi_{\mathrm{GCP}}}^{\mathrm{HS}}(a)$ is also positive. Using these information in our result we obtain,

$$
\lim _{T \rightarrow 0} \beta \hat{\mu}=\frac{c}{\phi_{\mathrm{GCP}}-\phi}
$$

with the numerical pre-factor given by,

$$
c=6 \pi \frac{\phi_{\mathrm{GCP}}}{\sigma^{3}} \frac{c_{2}}{c_{1}^{2}} \frac{113}{120}\left(y_{\phi_{\mathrm{GCP}}}^{\mathrm{HS}}(a)\right)^{3} .
$$

Using the numerical values of constants reported in [14] we find $c \sim 0.7$. A remarkable feature is that the scaling of the rigidity $\hat{\mu}$ found above Eq. (32) is exactly the same as that of the pressure $p$ found in [13] and [14]. We note that our result is different from that of Ref [10] which predicts somewhat stronger rigidity $\hat{\mu} \propto p^{3 / 2}$ based on an effective (yet microscopic) harmonic description developed for the inherent structures of the hardsphere glass.

The scaling (32) agrees with the plateau modulus $G_{\mathrm{p}}$ observed by MD simulations of the stress relaxation on the same system $[39,45]$. However the evaluation of the numerical factor $c$ (33) may be improved in several respects: 1) the approximation $y_{\phi}^{\mathrm{HS}}\left(\left|\mathbf{r}_{1}-\mathbf{r}_{2}\right|\right) \simeq y_{\phi}^{\mathrm{HS}}(a)$ can be avoided by doing numerical integrations 2 ) higher order corrections terms of the cage expansion due to renormalization of the potential $[13,14]$ can be considered 3 ) better evaluation of the 3-point correlation function $g_{3}\left(\mathbf{r}_{1}, \mathbf{r}_{2}\right)$ than the Kirkwood approximation may be considered. We have checked that the item 2) amounts to reduction of the value of $c$ by an amount of about $20 \%$ which will be reported elsewhere. The item 3) would be challenging but worthwhile.

It is interesting to compare the above result with some experimental observations of the rigidity of densely packed repulsive colloids [4] and emulsions [5, 6]. The experiments were performed at the room temperature which is actually a very low temperature for these systems. For example the reduced temperature can be estimated as $k_{\mathrm{B}} T / \varepsilon \sim 10^{-5}$ for the emulsions system [5, 6]. However the experimental data reveal presence of finite entropic rigidity which rapidly increase approaching the jamming density from below. A striking feature found by the experiment on the emulsion system is that simultaneous measurement of the pressure reveals that the pressure and the shear-modulus behave very similarly (See Fig. 3 of [5]). Thus it appears that the our theoretical result is consistent with the experiment.

In the present paper we have limited ourselves to the volume fractions $\phi<\phi_{\mathrm{GCP}}$, but it is straightforward to 
extended the present approach to the jammed region $\phi>\phi_{\mathrm{GCP}}$ concerning the systems of the soft repulsive contact potentials. It is important to note that for this class of systems one cannot rely on the usual picture of harmonic solids naively $[15,16,17]$. We will report the results elsewhere with detailed comparisons with the known results $[3,5,6,7,8,10,12]$.

\section{CONCLUSIONS}

In the present paper we first reviewed a microscopic approach to study the rigidity of structural glasses or the plateau modulus $G_{\mathrm{p}}$ of supercooled liquids based on the cloned liquid approach. Then we discussed how to extend the method, which has been limited to the cases of harmonic glasses, i. e. systems with analytic potentials, to the cases of the glassy repulsive contact systems like the hardsphere glasses or soft repulsive contact systems in the low temperature limit. We found the entropic rigidity of this class of systems exhibit divergent behaviour much as the pressure approaching the jamming density from below, which appear to be consistent with experimental observations on repulsive colloids and emulsions.

\section{ACKNOWLEDGMENTS}

The author thanks Marc Mézard, Satoshi Okamura, Anaël Lemaître, Hugo Jacquin and Francesco Zamponi for useful discussions. This work is supported by a Triangle de la physique grant number 117 "Intermittent response of glassy systems at mesoscopic scales", and Grant-in-Aid for Scientific Research (C) (50335337).

\section{REFERENCES}

1. A. Angell, K. L. Ngai, G. B. McKenna, P. F. McMillan, S. W. Martin, J. of App. Phys. 88, 3113 (2000).

2. E. R. Weeks, in "Statistical Physics of Complex Fluids", pp. 2-1 - 2-87, eds. S Maruyama \& M Tokuyama (Tohoku University Press, Sendai, Japan, 2007).

3. D. J. Durian, Phys. Rev. Lett. 75, 4780 (1995).

4. T. G. Mason and D. A. Weitz, Phys Rev. Lett. 75, 2770 (1995).

5. T. G. Mason, J. Bibette and D. A. Weitz, Phys Rev. Lett. 75, 2051 (1995).

6. T. G. Mason, Martin-D. Lacasse, Gary S. Grest, Dov Levine, J. Bibette, D. A. Weitz, Phys. Rev. E 56, 3150 (1997).

7. C. S. O'Hern, L. E. Silbert, A. J. Liu and S. R. Nagel, Phys. Rev. E 68, 011306 (2003).

8. M. Wyart, Ann. Phys. (Paris) 30, 1 (2005).

9. O. Dauchot, G. Marty, and G. Biroli, Phys. Rev. Lett. 95, 265701 (2005)

10. C. Brito and M. Wyart, Euro. Phys. Lett. 76, 149(2006).
11. L. Berthier and T. Witten, Euro. Phys. Lett. 8610001 (2009).

12. M. V. Hecke, J. Phys.: Condens. Matter, 22033101 (2010).

13. G. Parisi and F. Zamponi, Rev. Mod. Phys. 82, 789 (2010).

14. L. Berthier, H. Jacquin and Z. Zamponi, Phys. Rev. Lett. 106, 135702 (2011) and Phys. Rev. E 84, 051103 (2011).

15. C. F. Schreck, T. Bertrand, C. S. O'Hern, and M. D. Shattuck, Phys. Rev. Lett. 107, 078301 (2011).

16. M. Wyart, arXiv:1202.0259.

17. A. Ikeda, L. Berthier and G. Biroli, arXiv:1209.2814.

18. M. Otsuki and H. Hayakawa, Phys. Rev. E 86. 031505 (2012).

19. C. Coulais, R. P. Behringer and O. Dauchot, arXiv: 1202.5687.

20. P. Olsson and S. Teitel, Phys. Rev. Lett. 99, 178001 (2007)

21. T. Hatano, J. Phys. Soc. Jpn. 77 (2008) 123002.

22. M. Otsuki and H. Hayakawa, Prog. Theor. Phys. 121 (2009) 647.

23. A. Ikeda, L. Berthier and P. Sollich, Phys. Rev. Lett. 109, 018301 (2012).

24. M. Mézard and G. Parisi, Phys. Rev. Lett. 82, 747 (1999) and J. of Chem. Phys, 1111076 (1999).

25. B. Coluzzi, M. Mézard, G. Parisi and P. Verrochio, J. of Chem. Phys, 1119039 (1999).

26. L. Berthier, private communication.

27. T. R. Kirkpatrick and D. Thirumalai, Phys. Rev. Lett. 58, 2091 (1987), T. R. Kirkpatrick and P. G. Wolynes, Phys. Rev. B 36, 8552 (1987), T. R. Kirkpatrick, D. Thirumalai, and P. G. Wolynes, Phys. Rev. A 40, 1045 (1989).

28. G. Biroli and J. -P. Bouchaud, The Random First-Order Transition Theory of Glasses: a critical assessment in Structural Glasses and Supercooled Liquids: Theory, Experiment, and Applications Eds: P. G. Wolynes, V. Lubchenko, Wiley (2012), (preprint arXiv:0912.2542).

29. H. Yoshino and M. Mézard, Phys. Rev. Lett. 105, 015504 (2010).

30. H. Yoshino, J. Chem. Phys. 136, 214108 (2012).

31. F. H. Stillinger and T. A. Weber, Phys. Rev. A 25, 978 (1982).

32. B. Doliwa and A. Heuer, Phys. Rev. E 67, 031506 (2003).

33. R. Monasson, Phys. Rev. Lett. 75, 2847 (1995).

34. S. Franz and G. Parisi, Phys. Rev. Lett. 79, 2486 (1997).

35. J.-P. Hansen and I. R. Mcdonald, "Theory of simple liquids", Academic Press (2006).

36. D. R. Squire, A. C. Holt, and W. G. Hoover, Physica, 42, 388 (1969).

37. O. Farago and Y. Kantor, Phys. Rev. E 61, 2478 (2000).

38. J. L. Barrat, J. -N. Roux, J.-P. Hansen and M. L. Klein, Europhys. Lett. 7 (1988) 707.

39. S. Okamura, master-thesis, Osaka Univ. (2012).

40. H. Yoshino and A. Lemaître, in preparation.

41. S. Abraham and P. Harrowell, J. Chem. Phys. 137, 014506 (2012).

42. J. N. Roux, J. L. Barrat, and Hansen, J. of Phys.: Condensed Matter 1, 7171 (1989).

43. W. Götze and L. Sjögren, Rep. Prog. Phys. 55, 241 (1992).

44. G. Szamel and E. Flenner, Phys. Rev. Lett. 107, 105505 (2011).

45. S. Okamura and H. Yoshino, in preparation. 